\title{
Improved Design of Small Agricultural Machinery Transmission
}

\author{
WANG $\mathrm{Xi}^{1, \mathrm{a}}, \mathrm{GAO} \mathrm{YanYu}^{2, \mathrm{~b}}$, LI JianJun ${ }^{3, \mathrm{c} *}$ \\ ${ }^{1}$ Yunnan Agricultural University, Faculty of Mechanical and Electrical Engineering, \\ Kunming 650201,China \\ ${ }^{2}$ Yunnan Agricultural University, Faculty of Mechanical and Electrical Engineering, \\ Kunming 650201,China \\ ${ }^{3}$ Yunnan Agricultural University, Faculty of Mechanical and Electrical Engineering, \\ Kunming 650201,China \\ aemail: 3312561757@qq.com, bemail:928051487@qq.com \\ *Corresponding author: Li Jianjun (1970-), male, Anyue, Sichuan Province, associate professor, \\ mainly engaged in the research aspects of the automotive tractor
}

\section{Key words: Agricultural machinery; Gearbox; Constructional design}

Abstract: The transmission has a great relationship with the success of acceleration or deceleration and shift in agricultural machinery operations. The operating environment of agricultural machinery is complex and it is easy to cause damage to parts. In this paper, a kind of transmission for small agricultural machinery is designed. The length of the transmission rod can be stretched through a spiral compression and telescopic structure. The length of the transmission rod can be adjusted for operators with different arm lengths, and the comfort of the operators in farming can be guaranteed. The efficiency of farming is improved and the range of application of gearbox is improved effectively.

\section{Introduction}

The gearbox is mainly divided into manual and automatic. The manual gearbox is mainly composed of gears and shafts and generates variable speed torque by different gear combinations. The automatic transmission is composed of a hydraulic torque converter, planetary gears, a hydraulic variable pitch system and a hydraulic control system. The transmission variable torque is achieved through hydraulic transmission and gear combination.

At present, the shift levers of the existing gear transmissions for small agricultural machines are mostly of fixed length and cannot be adjusted in telescopic manner, and it is not possible to adjust the length of the shift levers for operators with different arm lengths. It greatly reduces the operator's comfort during farming and reduces the application of the gearbox. In addition, there is a lot of dust during farming, and the sealing performance between the shift lever and the gearbox is weak, which reduces the life of the gearbox.

\section{Structure and working principle of gearbox for small agricultural machinery}

\section{The whole frame}

The overall structure of the gearbox is shown in Figure 1. Mainly consists of transmission main body, sealing sleeve, connecting block, telescopic rod, hoop, pressure washer, oblique sleeve, 
inclined hole, knob, fastening bolt, grip, compression sleeve, and shift lever. Wherein: the upper surface of the transmission main body is provided with a sealing sleeve, the outer surface of the sealing sleeve is sleeved with a hoop, and the sealing sleeve is internally sleeved with a connecting block. An upper end surface of the connecting block is provided with a telescopic rod, and the outer surface of the lower side of the telescopic rod is tightly pressed with a pressing gasket, and the right side of the pressing gasket is provided with a barb. Inside the slanting sleeve, the gasket can be effectively pressed off, and the spacer is fixed on the right side of the pressing gasket. The pressing gasket is inserted into the inclined sleeve through the gasket fixing rod, and the right end surface of the pressing gasket is fixedly connected to the inclined sleeve. The outer surface sleeve of the slanting sleeve is sleeved with a pressing sleeve tightly pressed through an oblique hole, and a knob is fixedly connected to the upper end surface of the pressing sleeve. The interior of the knob is threadedly connected to the shift lever through a third threaded hole, and a first through hole is provided inside the upper right side of the knob. The knob is connected with a fastening bolt through the first through hole, the left side of the fastening bolt is threadedly connected to the gear shifting rod through the first threaded hole, the shift rod is arranged on the upper side of the oblique sleeve, and the grip is arranged on the upper side of the gear shift rod.

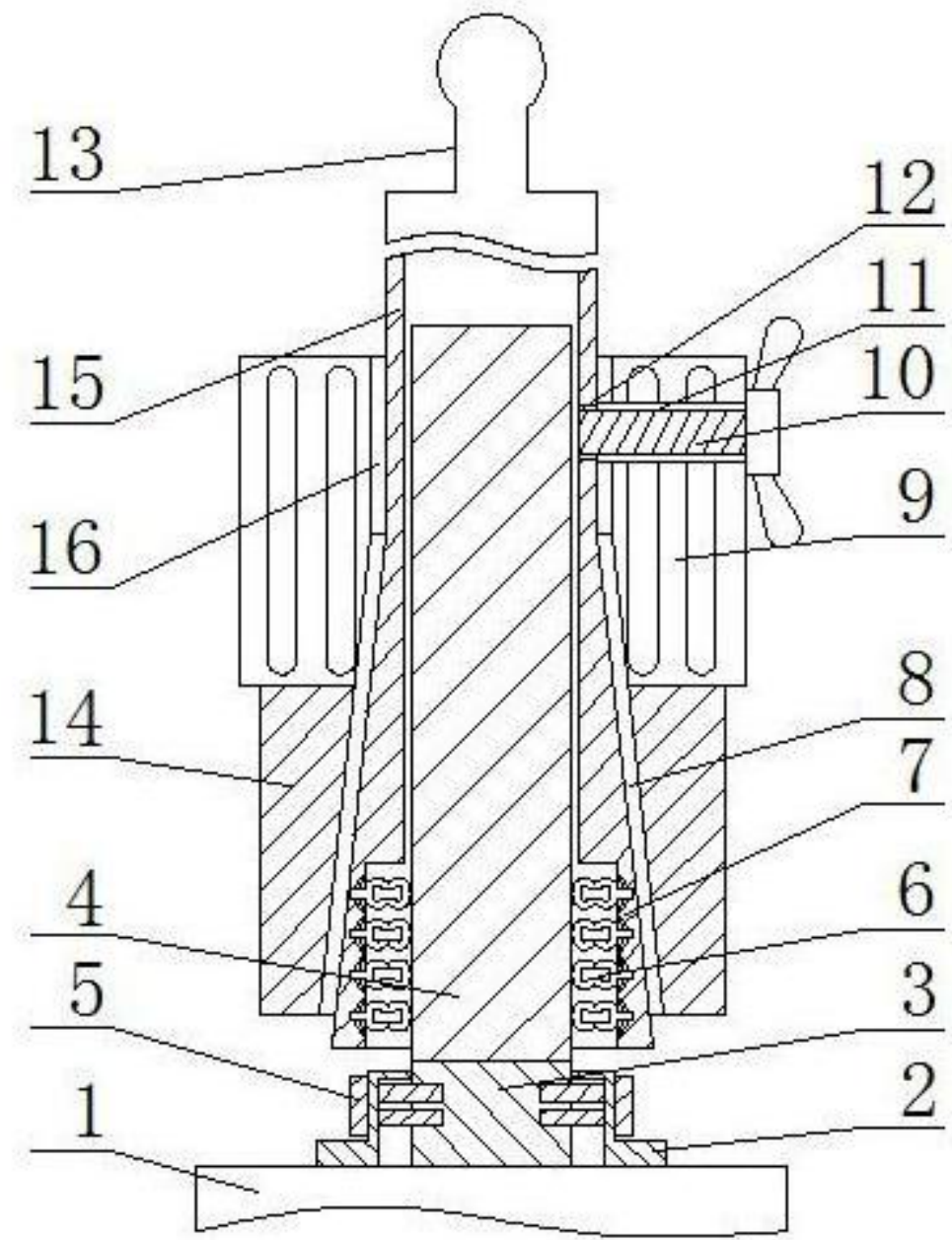

1.transmission main body 2.sealing sleeve 3.connecting block 4.telescopic rod 5.hoop 6. pressure washer 7.oblique sleeve 8.inclined hole 9.knob 10.fastening bolt 11.first through hole 12.first threaded hole 13.grip 14.compression sleeve 15.shift lever 16.third tapped hole

Figure 1 Gearbox integral structure diagram

\section{Principle of work}

When in use, pull the grip upwards and drive the shift lever to slide upwards on the outer 
surface of the telescopic rod. When the shift lever is moved to a suitable position, the knob is rotated clockwise, the knob is spirally moved downward through the third threaded hole, and the pressure sleeve is driven to move downwards. The compression sleeve gives the pressing force inside the oblique sleeve phase through the oblique hole, and drives the compression gasket on the internal surface of the oblique sleeve to press the outer surface of the extension rod. Rotate the fastening bolt clockwise, and tighten the bolt to lock the knob through the first threaded hole to realize the length extension and retraction of the shift lever. The length of the shift lever can be adjusted for different arm length operators. It ensures the operator's comfort when farming, improves the efficiency of farming, and effectively improves the application of the gearbox. Further clockwise rotation of the bolt, the bolt screw hole through the second hoop screw locking hoop pressing force given to the inner side of the sealing sleeve, drive block inserted inside the card slot. At the same time, the left end surface of the cleat clings to the inside of the slot, which effectively enhances the tightness of the seal sleeve and improves the life of the gearbox when used in a large amount of dust.

\section{Main parts introduction}

\section{Structure of connected block}

The connecting block is sleeved on the inside of the sealing sleeve, and the card slot is symmetrically arranged on both sides of the connecting block. The connecting block is connected with a clamping block through a slot, the right end surface of the clamping block is fixedly connected to the sealing sleeve, and the upper end surface of the connecting block is provided with a telescopic rod, and the left end surface of the clamping block is close to the inside of the clamping slot. Effectively enhances the tightness of the sealing sleeve and increases the life of the gearbox when used in a large amount of dust.
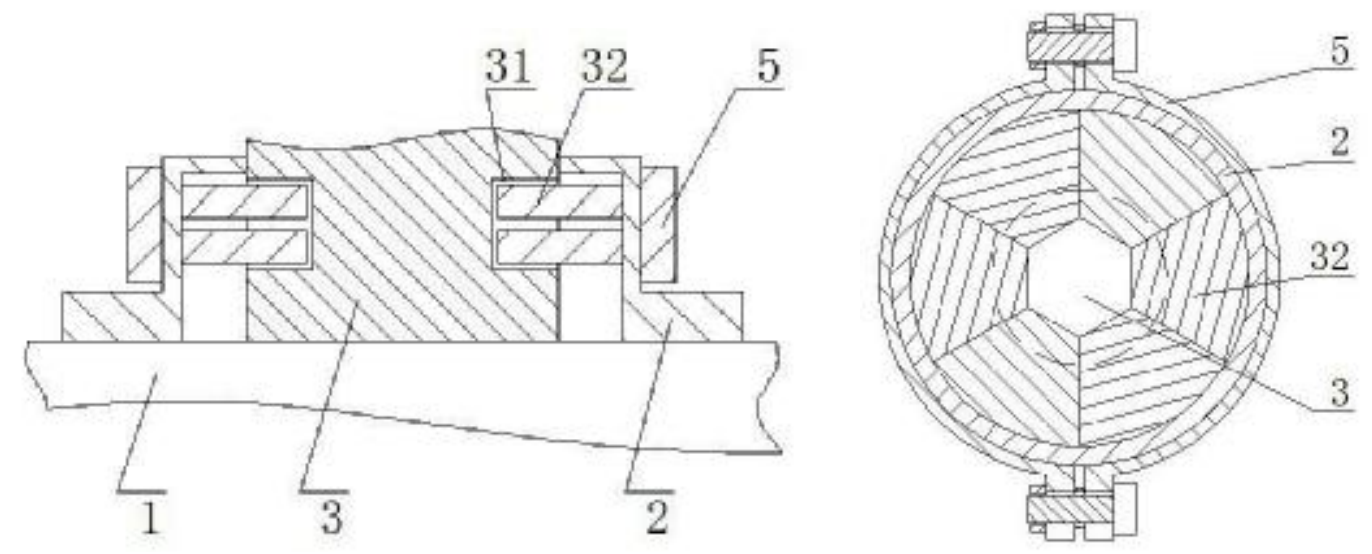
1. Transmission main body
2. sealing gland
3. link block
5. hoop 31.neck
32. block

Figure 2 Connecting block master, side-view diagram

\section{Structure of hoop}

The ferrule is attached to the outside surface of the gland and is a fastening member. A second through hole is symmetrically arranged on both sides of the hoop. A bolt is inserted through the second through hole, and the bolt is rotated clockwise. The bolt spirally locks the hoop through the second threaded hole, and the hoop gives a pressing force to the inside of the sealing sleeve. The middle surface of the bolt is sleeved with a spring washer through the third through hole. The spring washer is a washer with elasticity to prevent the bolt from loosening. The outer surface of the left 
side of the bolt is screwed with a non-slip nut through the second threaded hole. The anti-skid nut is a one-piece nut with a gasket on one side, and an anti-skid tooth pattern on the contact surface with the hoop, which effectively enhances the locking force of the anti-skid nut and ensures the sealing effect of the sealing sleeve.

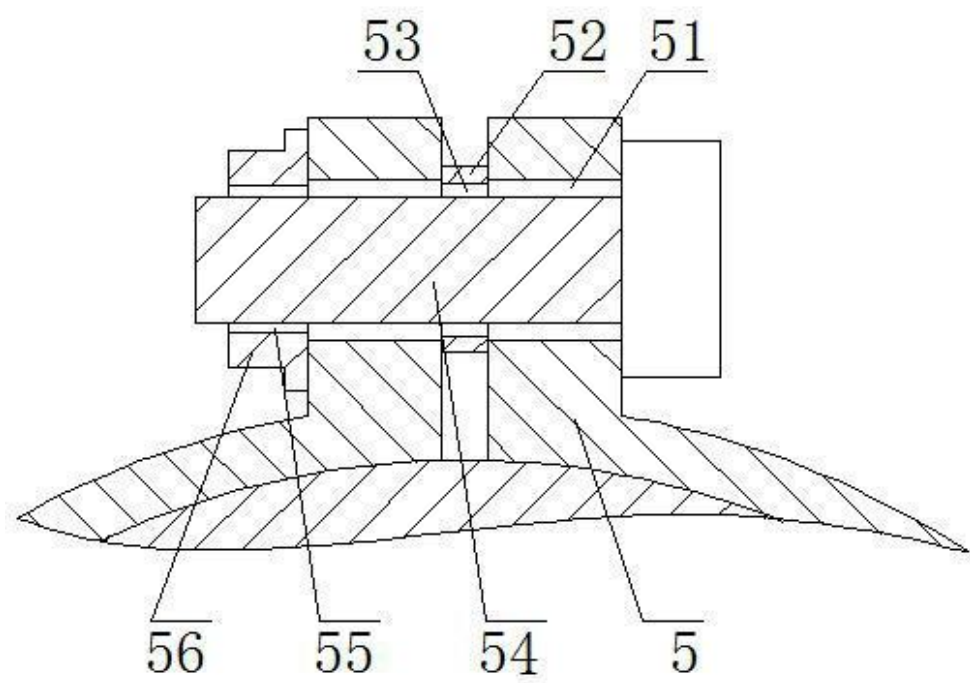

5.hoop 51.second through hole 52.spring washer 53.third through hole 54.bolt 55.second threaded hole 56. nut

Figure 3 The structure of the hoop

\section{Design of telescopic pole}

The outer surface of the telescopic rod is tightly pressed against the outer surface of the telescopic rod. The compression gasket is made of soft silicone material and its interior is a hollow design. When the slanting sleeve presses the compression washer inward, the left end of the compression washer is pressed against the outer surface of the telescopic rod. The soft silicone that compresses the gasket will be crushed and tightly fitted to the outer surface of the telescopic rod. Moreover, the silicone material has good friction performance, which can effectively prevent the telescopic rod and the compression sleeve from rotating and sliding.

\section{Conclusion}

The transmission gear for small agricultural machinery realizes the expansion and contraction of the gear lever by screwing the telescopic structure. The length of the shift lever can be adjusted for operators with different arm lengths, which ensures the operator's comfort during farming, improves the farming efficiency, and effectively improves the application range of the gearbox.

\section{References}

1. Y Huang, $\mathrm{H}$ Zhou, $\mathrm{J}$ Wang. Optimization and improvement of the transportation and transmission device of shrubs stubble machine. Journal of Forestry Engineering.

2. J Guo, N Yang, J Zhang. A Kind of BiCMOS Amplifier for Wireless Transmission Device Using in the Modern Farm Machinery. Journal of Agricultural Mechanization Research.

3. S Rahman, RK Varma, R Seethapathy.Novel Control of Grid Connected Photovoltaic (PV) Solar Farm for Improving Transient Stability and Transmission Limits Both During Night and Day. 21 St World Energy Congress. 